\title{
Comparison of Solution of a Class of Second Order Difference Equation
}

\author{
Y.Z. Qin \\ School of Mathematics and Statistics \\ Hechi University \\ Guang xi, Yizhou 546300, P. R. China
}

\begin{abstract}
Difference equations is a kind of powerful tool to investigate the rule of natural phenomena, such as, physical problems arising in a wide variety of applications. In this paper, we discuss the nodes of the solutions of a dass of second order difference equation.
\end{abstract}

Keywords-comparison theorem; difference equation; solution; nodes

\section{INTRODUCTION}

Difference equations is a kind of powerful tool to investigate the rule of natural phenomena, such as, physical problems arising in a wide variety of applications. More attentions are paid to difference equations [1-5]. Cheng and Cho [3] discussed the following second order difference equations.

$$
\begin{aligned}
& \Delta^{2} x(k-1)+p_{1}(k) x(k)=0, \\
& \Delta^{2} y(k-1)+p_{2}(k) y(k)=0,
\end{aligned}
$$

where $\mathrm{p}(\mathrm{k})$ is a real valued function defined on a set of consecutive integers to be specified later. Motivated by the results given in $[1,2,3,4,5]$, in this paper, we discuss nodes of solutions of the following second order difference equation

$$
\begin{aligned}
& \Delta^{2} x(k-1)+p_{1}(k) x^{2}(k)=0, \\
& \Delta^{2} y(k-1)+p_{2}(k) y^{2}(k)=0 .
\end{aligned}
$$

Suppose that $x(k)$ and $y(k), k \in I_{1}$, are respectively nontrivial solutions of the second order difference equations (1.1) and (1.2), and $p_{2}(k) y(k) \geq p_{1}(k) x(k)$ for all $k \in I_{3}$. If $x(k)$ has two consecutive nodes $a$ and $b$ in $\left[n_{0}, m\right]$, we prove that $y(k)$ has a node in $(a, b]$.

\section{RESULTS}

Throughout this paper, $\mathrm{N}$ denotes the set of natural numbers, $\mathrm{R}$ denotes the set of real numbers. Let $I_{1}=\left\{n_{0}, n_{0}+1, \cdots, m\right\}, \quad, \quad I_{2}=\left\{n_{0}, n_{0}+1, \cdots, m-1\right\}$

$I_{3}=\left\{n_{0}+1, n_{0}+2, \cdots, m-1\right\}, n_{0}, m \in \in_{\mathrm{N}}$.

Let $x$ be a real function defined on $I_{1}$. By straight line segments we join the points $(k, x(k)), k \in I_{1}$ to form a broken line. Then this broken line is the graph of a continuous function $x^{*}(t)$, such that $x^{*}(t)=x(t)$ for $k \in I_{1}$. The zeros of $x^{*}(t)$ are called the nodes of $x(t)$ For the convenience of description, we define a function by

$W(k)=x(k+1) y(k)-y(k+1) x(k), \forall k \in I_{2}$

We state the following theorems, which are similar to the results of Cheng and Cho [3].

Lemma 1. Let $(b-1), b \in I_{1}$. Suppose that $x$ and $y$ are real functions defined on $I_{1}, x(b-1)>0, y(b-1)>0$, and $x(k)$ has a node $\beta$ in $(b-1, \beta), y(k)$ has not node in $(b-1, \beta]$. Then $W(b-1)<0$.

Proof. Since $x(b-1)>0$ and $x(k)$ has a node $\beta$ in $(b-1, \beta), x(b)<0$.

If the function $y(k)$ has not node in $(b-1, b)$, then $y(b)>0$. Since $x(b-1)>0, x(b)<0, y(b-1)>0$,

$W(b-1)=x(b) y(b-1)-y(b) x(b-1)<0$.

If $y(k)$ has a node $\delta$ in $(b-1, b)$, Since $y(k)$ has not node in $(b-1, \beta]$, then $\delta>\beta>(b-1)$.

By the definitions of nodes and $\mathrm{W}$, we have

$$
\begin{aligned}
& W(b-1)=x(b) y(b-1)-y(b) x(b-1) \\
&=\left(\frac{x(b)}{x(b-1)}-\frac{y(b)}{y(b-1)}\right) x(b-1) y(b-1) \\
&=\left(\frac{b-\beta}{b-1-\beta}-\frac{b-\delta}{b-1-\delta}\right) x(b-1) y(b-1) \\
&=(b-(b-1-\delta)-(b-\delta)(b-1-\beta) \\
&=\quad x(b-1) y(b-1) \\
&=\frac{(\beta-\delta) x(b-1) y(b-1)}{(b-1-\beta)(b-1-\delta)} .
\end{aligned}
$$

Since $x(b-1)>0, y(b-1)>0$, and $\delta>\beta>(b-1)$, $W(b-1)<0$. 
Theorem 1. Let $x\left(n_{0}\right) \geq y\left(n_{0}\right)$ and $x\left(n_{0}+1\right) y\left(n_{0}\right)-y\left(n_{0}+1\right) x\left(n_{0}\right) \geq 0$. Suppose that $x(k)$ and $y(k), k \in I_{1}$, are positive solutions of the second order difference equations (1.1) and (1.2), respectively. If $p_{2}(k) y(k) \geq p_{1}(k) x(k)$ for $k \in I_{1}$, then $x(k) \geq y(k)$ for all $k \in I_{1}$.

Proof. Using (1.1), (1.2) and (2.1), we have

$$
\Delta W(k)=
$$

$x(k+2) y(k+1)-y(k+2) x(k+1)-x(k+1) y(k)+y(k+1) x(k)$ $=-x(k+1)[y(k+2)+y(k)]+y(k+1)[x(k+2)+x(k)]$

$$
\begin{gathered}
=-x(k+1)[y(k+2)-2 y(k+1)+y(k)] \\
+y(k+1)[x(k+2)-2 x(k+1)+x(k)] \\
=-x(k+1)\left[\Delta^{2} y(k)\right]+y(k+1)\left[\Delta^{2} x(k)\right] \\
=\left[p_{2}(k+1) y(k+1)-p_{1}(k+1) x(k+1)\right] x(k+1) y(k+1), \forall k \in I_{2},
\end{gathered}
$$

Since $p_{2}(k) y(k) \geq p_{1}(k) x(k)$, and $x(k)$ and $y(k)$ are positive for $k \in I_{1}, \Delta W(k) \geq 0$ for $k \in I_{2}$.

Furthermore, since $W\left(n_{0}\right)=x\left(n_{0}+1\right) y\left(n_{0}\right)-y\left(n_{0}+1\right) x\left(n_{0}\right) \geq 0$, we have

$$
W(k)=\frac{W\left(n_{0}\right)+\sum_{i=n_{0}}^{k} \Delta W(i)}{\geq 0, \forall k \in I_{3} .}
$$

Using (2.4), we get

$$
\begin{gathered}
\Delta\left(\frac{x(k)}{y(k)}\right)=\frac{x(k+1)}{y(k+1)}-\frac{x(k)}{y(k)} \\
=\frac{x(k+1) y(k)-y(k+1) x(k)}{y(k) y(k+1)} \geq 0, \quad \forall k \in I_{2} .
\end{gathered}
$$

By (2.5), the function $x(k) / y(k)$ is a nondecreasing function on $k \in I_{1}$. Since $x\left(n_{0}\right) \geq y\left(n_{0}\right)$, we have

$$
1_{\leq} \frac{x\left(n_{0}\right)}{y\left(n_{0}\right)} \frac{x\left(n_{0}+1\right)}{y\left(n_{0}+1\right)} \leq \ldots \leq \frac{x(m)}{y(m)} .
$$

Obviously, $x(k) \geq y(k)$ for all $k \in I_{1}$, which is the desired relationship. Theorem 2. Suppose that $x(k)$ and $y(k), k \in I_{1}$, are respectively nontrivial solutions of the second order difference equations (1.1) and (1.2), and $p_{2}(k) y(k) \geq p_{1}(k) x(k)$ for all $k \in I_{3}$. If $x(k)$ has two consecutive nodes $a$ and $b$ in $\left[n_{0}, m\right]$, then $y(k)$ has a node in $(a, b]$.

Proof. Let $\mathrm{n}_{0} \leq \mathrm{n}_{\mathrm{J}}-1 \leq a<\mathrm{n}_{\mathrm{J}} \leq \mathrm{n}_{\mathrm{K}} \leq b \leq \mathrm{n}_{\mathrm{J}}-1 \leq m$,
$\mathrm{n}_{\mathrm{J}}, \mathrm{n}_{\mathrm{K}} \in I_{1}$. Since $a$ and $b$ are two consecutive nodes of $x(k)$,without loss of generality we can assume that the broken line $x^{*}(t)>0$ for all $t \in\left[\mathrm{n}_{\mathrm{J}}, \mathrm{n}_{\mathrm{K}}\right] \subset(a, b]$. Obviously, $x\left(\mathrm{n}_{\mathrm{J}}-1\right)<0, x\left(\mathrm{n}_{\mathrm{J}}\right)>0, x\left(\mathrm{n}_{\mathrm{K}}\right)>0, x\left(\mathrm{n}_{\mathrm{K}}+1\right)<0$.

If we assume that there is not node of $y(k)$ in $(a, b]$, then the sign of $y^{*}(t)$ doesn't change in $(a, b]$. Without loss of generality we assume that $y^{*}(t)>0$ in $(a, b]$, then $y\left(\mathrm{n}_{\mathrm{J}}\right)>0, y\left(\mathrm{n}_{\mathrm{K}}\right)>0$. Using the method of proof in le mma 1 , we can obtain that

$W\left(\mathrm{n}_{\mathrm{J}}-1\right)=x\left(\mathrm{n}_{\mathrm{J}}\right) y\left(\mathrm{n}_{\mathrm{J}}-1\right)-y\left(\mathrm{n}_{\mathrm{J}}\right) x\left(\mathrm{n}_{\mathrm{J}}-1\right)>0$, and

$$
W\left(\mathrm{n}_{\mathrm{K}}\right)=x\left(\mathrm{n}_{\mathrm{K}}+1\right) y\left(\mathrm{n}_{\mathrm{K}}\right)-y\left(\mathrm{n}_{\mathrm{K}}+1\right) x\left(\mathrm{n}_{\mathrm{K}}\right)>0 .
$$

On the other hand, by Theorem 1, we have

$$
\Delta W(k)=\left[p_{2}(k+1)-p_{1}(k+1)\right] x(k+1) y(k+1), \forall k \in I_{2}(
$$

From (2.7) and (2.9), we have

$$
W\left(\mathrm{n}_{\mathrm{K}}\right)_{=} W\left(\mathrm{n}_{\mathrm{J}}-1\right)+\sum_{j=n_{J}}^{\mathrm{n}_{\mathrm{K}}} \Delta W(j)>0,
$$

There is a contradiction between (2.8) and (2.10). So $y(k)$ has a node in $(a, b]$.

\section{SUMMARY}

Difference equations is a kind of powerful tool to investigate the rule of natural phenomena, such as, physical problems arising in a wide variety of applications. In this paper, we discuss the solutions of a class of second order diffe rence equation.

Let $\quad x\left(n_{0}\right) \quad \geq \quad y\left(n_{0}\right) \quad$ and $x\left(n_{0}+1\right) y\left(n_{0}\right)-y\left(n_{0}+1\right) x\left(n_{0}\right) \geq 0$. Suppose that $x(k)$ and $y(k), k \in I_{1}$, are positive solutions of the second order difference equations (1.1) and (1.2), respectively. If $p_{2}(k) y(k) \geq p_{1}(k) x(k)$ for $k \in I_{1}$, then $x(k) \geq y(k)$ for all $k \in I_{1}$.

Suppose that $x(k)$ and $y(k), k \in I_{1}$, are respectively nontrivial solutions of the second order difference equations (1.1) and (1.2), and $p_{2}(k) y(k) \geq p_{1}(k) x(k)$ for all $k \in I_{3}$. If $x(k)$ has two consecutive nodes $a$ and $b$ in $\left[n_{0}, m\right]$, then $y(k)$ has a node in $(a, b]$.

\section{ACKNOWLEDGEMENT S}

This research was supported by Key Projection of Hechi University of China (No. 2014ZD-N003). 


\section{REFERENCES}

[1] E. J. Moulton, A theorem in difference equations on the alternation of nodes of linearly independent solutions. Annals of Mathematics, 13, pp. 137-139, 1911-1912.

[2] E. Makai, On a monotonic property of certain sturm-liouville functions. Acta Mathematica Acaiemiae Scientiarum Hungaricae, 3, pp. $165-172,1952$.

[3] S. S. Cheng and A. M. Cho, Convexity of nodes of discrete sturmliouville functions. Hokkaido Mathematical Journal, 11, pp. 8-14, 1982.

[4] S. S. Cheng, A discrete analogue of the inequality of lyapunov. Hokkaido Mathematical Journal, 12, pp. 105-112, 1983.

[5] B. D. Pachpatte, On lyapunov-type inequalities for certain higher order differential equations. Journal of mathematical analysis and applications, 195, pp. 527-536, 1995 